\title{
Caracterización de los comportamientos de riesgo para la salud en adolescentes de una escuela rural de Cañar
}

\section{Characterization of health risk behaviors in adolescents of a rural school in Cañar}

\author{
Xavier Rodrigo Yambay Bautista ${ }^{1}$, Andrés Leonardo Vázquez Cardenas ${ }^{1}$, Martín Adrián Contreras Sanango ${ }^{1}$ y \\ Jossette Rossemary Tello Riofrio ${ }^{2}$ \\ ${ }^{1}$ Universidad Católica de Cuenca \\ ${ }^{2}$ Hospital Aida León, Girón \\ *xyambayb@ucacue.edu.ec
}

DOI: https://doi.org/10.26871/killkana_salud.v3i3.525

\begin{abstract}
Resumen
Introducción: Los problemas que afectan a los adolescentes están interrelacionados y se asocian a una tendencia creciente a caer en conductas de riesgo que ponen en peligro su salud. Objetivo: Caracterizar los comportamientos que ponen en riesgo la salud de adolescentes escolarizados en una institución educativa rural de Cañar; determinando la prevalencia y particularidades sociodemográficas de los mismos. Materiales y métodos: Se trata de un estudio cuantitativo, no experimental, descriptivo y transversal en el que participaron la totalidad de adolescentes, hombres y mujeres entre 14 y 18 años ( $\mathrm{n}=137)$, que asisten a una escuela rural ubicada en la parroquia Ducur del cantón Cañar. Los datos fueron procesados empleando el paquete estadístico Statistical Package for the Social Sciences (SPSS) versión 24.0. El instrumento utilizado fue un cuestionario de aplicación anónima, cuidando los aspectos éticos y legales, elaborado y validado con una fiabilidad de consistencia interna de 0,72 (alfa de Cronbach) y que consta de 35 preguntas distribuidas en 5 dimensiones. Resultados: El 52.6\% de los participantes se encontró en un rango de edad entre 14 y 16 años, siendo en su mayoría mujeres $(68,6 \%)$; el $95.6 \%$ de los encuestados pertenecen al grado de bachillerato y el $38.7 \%$ vive con sus dos padres, el resto vive solo con un progenitor o con otros familiares. La prevalencia hallada para la dimensión violencia fue de $7.3 \%$; conductas sexuales de riesgo $16.8 \%$; consumo de sustancias $18.2 \%$; conductas suicidas $28.4 \%$; y, conductas alimentarias de riesgo y sedentarismo $43.1 \%$. Conclusiones: Los comportamientos de riesgo se caracterizan por su influencia adversa en el desarrollo bio-psico-social del adolescente. Prevalecen en la población estudiada las conductas alimentarias de riesgo y los comportamientos suicidas.
\end{abstract}

Palabras clave: adolescente, comportamiento, conducta, riesgo.

\begin{abstract}
Introduction: Problems affecting adolescents are interrelated and are associated with a growing tendency to fall into risky behaviors that endanger their health. Objective: Characterize the behaviors that put at risk the health of adolescents enrolled in a rural educational institution in Cañar; determining the prevalence and sociodemographic particularities of them. Materials and methods: This is a quantitative, non-experimental, descriptive and cross-sectional study in which all adolescents, men and women between 14 and 18 years old $(n=137)$, who attend a rural school participated located in the Ducur parish of the Cañar canton. The data were processed using the Statistical Package for the Social Sciences (SPSS) version 24.0 statistical package. The instrument used was a questionnaire of anonymous application, taking care of ethical and legal aspects, developed and validated with a reliability of internal consistency of 0.72 (Cronbach's alpha) and consisting of 35 questions distributed in 5 dimensions. Results: $52.6 \%$ of the participants were between 14 and 16 years old, mostly women (68.6\%); $95.6 \%$ of respondents belong to the baccalaureate degree and $38.7 \%$ live with their two parents, the rest live only with a parent or with other relatives. The prevalence found for the violence dimension was $7.3 \%$; $16.8 \%$ risk sexual behaviors; substance use $18.2 \%$; suicidal behaviors $28.4 \%$; and, risk eating behaviors and sedentary lifestyle $43.1 \%$. Conclusions: Risk behaviors are characterized by their adverse influence on the adolescent's bio-psycho-social development. Risk eating behaviors and suicidal behaviors prevail in the study population.
\end{abstract}

Keywords: adolescent, behavior, conduct, risk. 


\section{Introducción}

Abordar los temas relacionados con la adolescencia resulta siempre una tarea apasionante. La literatura existente propone que la adolescencia constituye un periodo de transición entre el niño y el adulto, marcada por innumerables cambios en la esfera biopsicosocial del individuo que contribuyen a la construcción de su personalidad, siendo importante en el proceso del desarrollo humano entre los 10 y los 19 años, en donde nunca desaparece la infancia, así como nunca se accede a una madurez absoluta. ${ }^{1-3}$

Los cambios biológicos y fisiológicos en los adolescentes incluyen el desarrollo de los órganos genitales, cambio de voz y crecimiento de vellos. Por su parte, los cambios psicológicos tienen que ver con el desarrollo mental, moral, la búsqueda de identidad y construcción del auto-concepto configurado con la autoimagen global; mientras que los cambios en el ámbito social implican cierto distanciamiento del contexto familiar, evidenciándose un apego significativo al grupo de amigos, donde los adolescentes buscan la confirmación de su identidad, la posibilidad de explorar nuevas relaciones sociales, y el apoyo y comprensión de otras personas que están atravesando una etapa evolutiva similar a la suya. ${ }^{4}$

Los problemas de salud prioritarios que afectan a los adolescentes están interrelacionados y se asocian a una tendencia creciente a caer en conductas de riesgo que ponen en peligro su salud, por lo que se requiere de acciones inmediatas e integradas. ${ }^{3,5}$ Diversos autores definen a las Conductas de Riesgo (CR), como aquellas actividades capaces de incrementar las posibilidades de deteriorar el estado de salud de una persona por morbilidad o mortalidad. ${ }^{6}$ Estos comportamientos de riesgo están en afinidad con la violencia o agresiones, consumo de sustancias, comportamiento suicida, conductas alimentarias de riesgo (CAR) y conductas sexuales de riesgo, que pueden terminar en múltiples problemas de salud pública como Infecciones de Transmisión Sexual (ITS); embarazos no deseados; los trastornos de los hábitos alimentarios; problemas de salud mental; violencia; abuso de sustancias psicotrópicas; accidentes de tránsito y/o suicidios. ${ }^{5}$

En este contexto y dada la importancia de la problemática que no ha sido estudiada a profundidad en las zonas rurales de la provincia del Cañar, surge la necesidad de desarrollar esta investigación, con referencia como punto de partida la información de adolescentes que estudian en una institución pública de la parroquia Ducur, mediante la determinación de la prevalencia y la caracterización de los comportamientos que ponen en riesgo la salud de los adolescentes escolarizados; que permitan a futuro tomar acciones encaminadas a disminuir el nivel de riesgo de estas conductas, que sin duda acarrean consecuencias que se convierten en grandes problemas de salud pública.

\section{Marco Teórico}

La adolescencia constituye una etapa clave del desarrollo humano que abarca las edades comprendidas entre los $10 \mathrm{y}$
19 años, caracterizada por una agudización en las contradicciones sobre la dinámica de las relaciones internas que terminan en la adquisición de estilos de vida que pueden ser saludables o problemáticos. ${ }^{1,7}$ En este período, el adolescente está implicado en un proceso de consolidación de su identidad y la familia tiene un particular significado, así como también su red social o grupo de iguales que llegan a convertirse en el principal apoyo del adolescente. ${ }^{4}$

La Organización Panamericana de la Salud (OPS) estima que alrededor de 70.000 adolescentes entre 10 y 19 años mueren cada año en la región de las Américas. De ellos 20.000 fallecen a causa de homicidios, suicidios y accidentes de transporte terrestre, convirtiéndose en las principales causas de muerte entre los adolescentes de la región, con tasas de mortalidad sistemáticamente más elevadas en el sexo masculino; resalta además que la mayoría de los hábitos perjudiciales para la salud se adquieren durante la adolescencia, lo que trae consigo problemas de salud en la edad adulta, y agrega una carga económica evitable a los sistemas de salud. ${ }^{5,8,9}$

El Ecuador no escapa a la realidad de la región ya que durante el año 2015 se registraron a los accidentes de tránsito, las lesiones auto-infligidas y los homicidios dentro de las principales causas de muerte en jóvenes de 10 a 19 años, donde fallecieron 634 adolescentes por estas causas. ${ }^{5}$ La Encuesta Nacional de Salud y Nutrición (ENSANUT-ECU 2012), describe estadísticas de algunas conductas que ponen en riesgo la salud del adolescente, con una prevalencia del consumo de tabaco del $28,4 \%$ y del $45,6 \%$ en el consumo de bebidas alcohólicas. En cuanto a la experiencia sexual, un 39,2\% de las mujeres encuestadas ya ha tenido relaciones sexuales; reportándose además que hacer dieta para adelgazar es una conducta practicada por el 4,8\% de adolescentes entre 10 a 19 años con predominio en las mujeres. ${ }^{10,11}$

Argaez et al. ${ }^{12}$ cita a varios autores para definir y caracterizar a las conductas de riesgo como: "Aquellas acciones voluntarias o involuntarias, realizadas por el individuo o comunidad, que pueden llevar a consecuencias nocivas. Ejemplos de estas son: las conductas delictivas y la conducta sexual de riesgo, el consumo de alcohol, tabaco y otras drogas"; a esta lista se suman el comportamiento suicida y las conductas alimentarias de riesgo. Ordoñez y Barros ${ }^{13}$ demuestran que un estilo de crianza basado en la autonomía y democracia puede influir en problemas conductuales en adolescentes.

Otro aspecto importante destacado por Villareal et al. ${ }^{4}$ es el entorno escolar del adolescente, en donde las relaciones entre familia y escuela comprenden desde los contactos más o menos ocasionales, hasta la colaboración conjunta en ciertos aspectos, o la decisión compartida sobre cuestiones relativas al funcionamiento del adolescente en la escuela. En este contexto, un tema de gran importancia muy relacionado con la escuela y la familia, es el comportamiento violento que algunos adolescentes muestran hacia sus compañeros y que en gran medida estas conductas de violencia 
confluyen en la formación de pandillas, bandas o naciones; peleas o riñas con otros jóvenes dentro y fuera de la escuela; robos; amenazas y heridas con armas, entre otros.

Por su parte, el consumo de sustancias en la adolescencia se ha convertido en un problema de salud pública de tendencia creciente a nivel mundial, con alto impacto en el desarrollo biopsicosocial del adolescente al ser un grupo neurobiológicamente vulnerable, que puede tener como desenlace un trastorno por consumo de sustancias. Por ejemplo, se reporta una prevalencia de $59,3 \%$ para el consumo de alcohol en adolescentes de una secundaria en México, estimando como un factor importante la disponibilidad y accesibilidad a la venta de alcohol alrededor de la institución educativa. ${ }^{14,15}$ Del mismo modo Da Silva ${ }^{16}$ cita a otros autores para explicar las manifestaciones y consecuencias del consumo de tabaco que a veces se asocia al consumo de alcohol, transformándose en un estimulante para la adopción de otros comportamientos de riesgo, entre ellos, la participación en el acoso escolar (violencia). Los resultados de un estudio realizado en Costa Rica por Méndez $\mathrm{z}^{17}$ aseveran que "una mayor relación con los padres no solamente puede disminuir los niveles de prevalencia de consumo, sino que también disminuye la posibilidad de exposición al consumo de sustancias", lo cual reduce el nivel de curiosidad y la necesidad de recurrir a espacios donde sea mayor la oferta de sustancias.

Las conductas sexuales de riesgo para la salud de los adolescentes, descritas por la literatura científica incluyen: inicio de la actividad sexual (48\% a los 15 años); cambios frecuentes de parejas (50,5\% de los adolescentes); relaciones sexuales sin protección (48\%); atracción y orientación familiar; pagar para mantener relaciones sexuales; recibir dinero u otra clase de dádivas por tener relaciones sexuales. Las consecuencias de estas prácticas sexuales se traducen en embarazos no deseados; riesgo de parto prematuro; nacimiento de niños con bajo peso al nacer; infertilidad y riesgo de cáncer y VIH, debido a la falta de tratamiento a largo plazo de las infecciones de transmisión sexual. 5, 7,18

A nivel mundial y en especifico en la región de las Américas, las tasas de suicidio en adolescentes de 10 a 19 años han ido en constante aumento, lo cual es preocupante para la salud pública. En el Ecuador se registraron 221 defunciones atribuidas a lesiones auto-infligidas/suicidios con una ligera prevalencia en los hombres y en el grupo de edad de 15 a 19 años. ${ }^{8}$ Con respecto al intento de suicidio, Silva ${ }^{19}$ describe en una muestra de adolescentes escolarizados de Chile, una prevalencia del 14,3\% asociada a variables como: sexo femenino, la ausencia de padres en el grupo familiar, el intento de suicidio en alguno de los padres, consumo de tabaco, alcohol o drogas y la ideación suicida.

Los trastornos de la conducta alimentaria son cada vez más frecuentes en la población, y afectan de forma desproporcional a las adolescentes mujeres e incluyen la anorexia nerviosa, la bulimia nerviosa y otros comportamientos no especificados (comer compulsivamente, consumo de co- mida chatarra, entre otros). El riesgo para presentar este tipo de trastornos se observó en el 30,1\% de estudiantes de una secundaria en Bogotá. Antes se pensaba que estos comportamientos aparecían solo en las sociedades de alto nivel económico; sin embargo, en los últimos años se han reportado casos en todos los estratos socioeconómicos y étnicos de la región de las Américas. ${ }^{5,20}$

Bajo estos conceptos se llevó a cabo el presente estudio con el objeto de identificar la prevalencia de las conductas de riesgo para la salud del adolescente en una institución educativa rural de la parroquia Ducur del cantón Cañar. Del mismo modo se detallan los parámetros sociodemográficos de los adolescentes que acuden a esta unidad educativa.

\section{Metodología}

Se trata de una investigación cuantitativa que contó con un diseño no experimental, transversal y descriptivo. Con una muestra no probabilística de selección por voluntarios $(n=137)$ en edades comprendidas entre 14 y 18 años pertenecientes a una institución educativa rural en la parroquia Ducur del cantón Cañar, ubicada en la zona costanera de la provincia del Cañar; con la participación de 94 mujeres y 43 hombres estudiantes de décimo de básica y de primero, segundo y tercero de bachillerato. Se aplicó la técnica de la encuesta, en la que se propusieron interrogantes que coadyuvaron con la identificación de los comportamientos de riesgo que presentan los adolescentes, con el cuidado de la confiabilidad y confidencialidad de la información, pues el proceso se aplicó de una forma anónima, previo a la firma del consentimiento informado de los representantes de los participantes y la autorización de los directivos de la unidad educativa.

El instrumento fue elaborado en base a la Guía para medir comportamientos de riesgo en jóvenes, ${ }^{21}$ misma que organiza dimensiones e indicadores de riesgo para la salud de los adolescentes a través de un enfoque multidisciplinario, siendo validada con una puntuación de 0,72 según la escala Alpha de Cronbach. El cuestionario, consta de dos partes distribuidas de la siguiente manera:

- Sección A: datos de identificación (sociodemográficos)

- Sección B: 35 preguntas dicotómicas (SI o NO) organizadas en 5 dimensiones a saber: Conductas violentas (preguntas 1 a 7); Consumo de sustancias (preguntas 8 a 16); conductas sexuales (preguntas 17 a 26); conductas suicidas (preguntas 27 a 29); y, trastornos de la conducta alimentaria y sedentarismo (preguntas 30 a 35). Cada respuesta $\mathrm{SI}=1$ punto y cada respuesta NO $=0$ puntos; excepto en las preguntas 24,30 y 34 donde el puntaje se invierte.

Los resultados se catalogaron en función del puntaje alcanzado por los encuestados en cada dimensión y los datos fueron procesados en el paquete estadístico Statistical Package for the Social Sciences (SPSS) versión 24.0. 


\section{Resultados}

Participaron 137 adolescentes que estudian en una institución pública rural de Cañar, 43 (31,4\%) hombres y 94 $(68,6 \%)$ mujeres; en edades de 17 a 18 años, pertenecientes al décimo de básica $(4,4 \%)$ y al bachillerato $(95,6 \%)$. La distribución y caracterización (variables sociodemográficas) de los adolescentes se presenta en la tabla 1. En lo referente a la estructura familiar de los adolescentes encuestados el $38,7 \%$ vive con sus dos progenitores; el $38,6 \%$ con un solo progenitor $(26,2 \%$ solo con madre; $10,3 \%$ solo con padre) y el $22,7 \%$ restante convive con otros familiares.

Tabla 1. Distribución de la muestra por sexo y rango de edad según la estructura familiar

\begin{tabular}{|c|c|c|c|c|c|}
\hline & \multicolumn{2}{|c|}{14 a 16 años } & \multicolumn{2}{|c|}{17 a 18 años } & \multirow[t]{2}{*}{ Total } \\
\hline & $\mathrm{H}$ & $M$ & $\mathrm{H}$ & $\mathrm{M}$ & \\
\hline Mis 2 padres & 13 & 14 & 11 & 15 & 53 \\
\hline Sólo con madre & 4 & 17 & 6 & 11 & 38 \\
\hline Sólo con padre & 3 & 5 & 2 & 5 & 15 \\
\hline Otros familiares & 2 & 14 & 2 & 13 & 31 \\
\hline Total & \multicolumn{2}{|c|}{72} & \multicolumn{2}{|c|}{65} & 137 \\
\hline
\end{tabular}

En la determinación de la prevalencia de conductas de riesgo para la salud de los adolescentes se evaluaron cada una de las cinco dimensiones por separado, identificándose con una mayor prevalencia a las conductas alimentarias de riesgo y sedentarismo (43,1\%); seguida de los comportamientos suicidas con $28,4 \%$; el consumo de sustancias ocupa el tercer lugar con una prevalencia del 18,2\%; a continuación las conductas sexuales de riesgo tienen una prevalencia de $16,8 \%$; y, finalmente las conductas violentas están presentes en el 7,3\% de los adolescentes encuestados (figura 1).

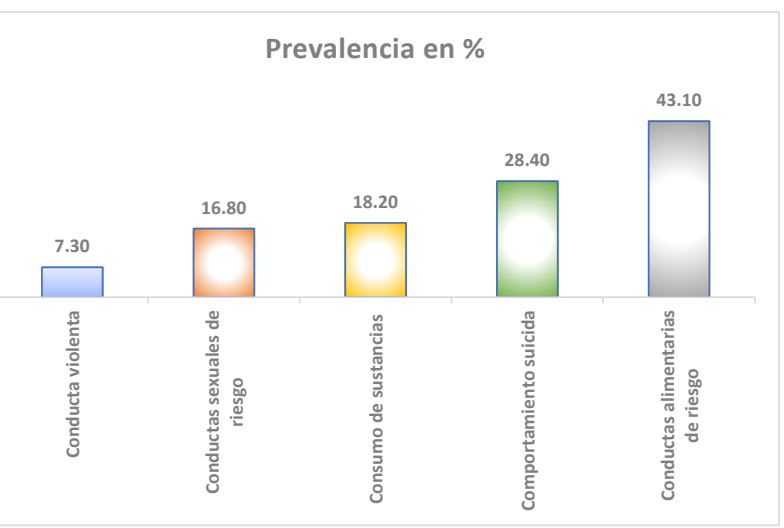

Fig. 1. Conductas de riesgo en adolescentes de una escuela rural de Ducur, Cañar

En la figura 2 se muestra la prevalencia de las conductas de riesgo para la salud en adolescentes, según el número de estudiantes que presentaron o no comportamientos de riesgo de acuerdo a las dimensiones estudiadas.
Conductas de riesgo para la salud adolescentes

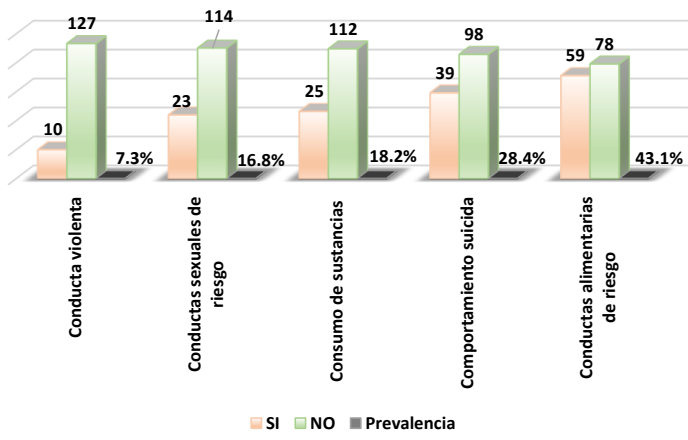

Fig. 2. Prácticas de riesgo en adolescentes escolarizados, según el número de estudiantes

\section{Discusión}

Los resultados de esta investigación en 137 adolescentes que estudian en una escuela pública rural de la zona costanera de la provincia del Cañar demuestran datos trascendentes en cuanto a la estructura familiar de los participantes. Solo el $38,7 \%$ vive dentro de una familia biparental mientras el $61,3 \%$ hace parte de familias fraccionadas (monoparentales o viven con otros familiares); esto contrasta con la información publicada por Villareal et $a l .{ }^{4}$ cuando destaca que: "En México, aunque la familia nuclear sigue representando al $64 \%$ del total, las familias sin hijos, así como los hogares unipersonales, van cobrando protagonismo".

En lo relacionado a la prevalencia de los comportamientos de riesgo, los hallazgos registrados corresponden a un porcentaje importante $(43,1 \%)$ en la dimensión conducta alimentaria y sedentarismo (afín con los estilos de vida de los adolescentes). Este porcentaje es elevado al comparar con el estudio de Fajardo et al..$^{20}$ que coloca la prevalencia de riesgo de trastornos alimentarios en el 30,1\% con mayor predominio en mujeres que en varones, aunque el estado nutricional en la mayoría se encontraba normal.

La prevalencia del comportamiento suicida encontrada en este trabajo fue del $28,4 \%$, porcentaje mayor a lo que se reporta en otros estudios como el de Silva ${ }^{19}$ en Chile, quien establece la prevalencia del intento de suicidio en un $14,3 \%$, y presenta como variables estadísticamente asociadas al sexo femenino, la ausencia de padres en el grupo familiar, el intento de suicidio en alguno de los padres, consumo de tabaco, alcohol o drogas, entre otras.

El consumo de sustancias revela que el $18,2 \%$ de los adolescentes que participaron en este estudio han probado alguna vez alcohol, tabaco u otras drogas; lo que demuestra una conducta de menor prevalencia si se consideran otras investigaciones a nivel internacional que determinan prevalencias de alrededor del $40 \%$ para consumo de alcohol; $7,7 \%$ para consumo de tabaco y $5 \%$ para drogas, principalmente la marihuana. ${ }^{2,7,17}$

Para las conductas sexuales de riesgo, en términos generales la prevalencia en la población estudiada fue de 
$16,8 \%$, tomando en cuenta sobre todo el inicio de la actividad sexual y los métodos de protección utilizados. Con este argumento se identificaron porcentajes relativamente similares en otros estudios internacionales que reportan $14 \%$ de adolescentes que empezaron su actividad sexual y $19 \%$ manifiesta haber mantenido relaciones sexuales no protegidas; además señalan al cambio frecuente de parejas y al desconocimiento como factores predisponentes para la aparición de infecciones de transmisión sexual ${ }^{7,18}$

Finalmente, la violencia es la dimensión con menor prevalencia $(7,3 \%)$ en los adolescentes estudiados, porcentaje de características similares a los publicados por varios investigadores citados por Villareal $^{4}$ ya que cifras cercanas al $7 \%$ denotan actos violentos reconocidos por los propios victimarios; mientras que un promedio del $21 \%$ de estudiantes secundarios afirman haber sufrido algún tipo de violencia o maltrato en la escuela. Respecto a la brecha de los valores antes mencionados, los autores explican que este fenómeno puede ser posible debido a que un solo alumno es capaz de agredir a varios.

\section{Conclusiones}

Luego de haber realizado el abordaje teórico, la aplicación del instrumento y el análisis de resultados acerca de los adolescentes y las conductas de riesgo que pueden afectar su salud, se concluye que:

La adolescencia es una etapa de cambios en el aspecto biopsicosocial, por lo que esta población constituye un grupo vulnerable con mayor propensión a la adquisición consciente o inconsciente de conductas o comportamientos de riesgo para la salud. Esta investigación tamizó conductas de riesgo en 137 adolescentes escolarizados de una unidad educativa pública rural de la zona costanera de la Provincia del Cañar, Ecuador. Se pudo caracterizar a los adolescentes y las conductas que ponen en riesgo su entorno biopsicosocial, encontrando que 7 de cada 10 participantes son mujeres; la mayoría cursando el bachillerato, entre los 17 y 18 años.

La información recopilada puso de manifiesto que un poco más de la mitad de adolescentes encuestados provienen de un hogar con una estructura familiar fragmentada, ya que viven solo con uno de los padres o con otro familiar. Los riesgos a los que principalmente están expuestos los adolescentes se congregan en cinco aspectos enfocados en las siguientes dimensiones descritas por orden de prevalencia: (i) trastornos de la conducta alimentaria y sedentarismo; (ii) comportamientos suicidas; (iii) consumo de sustancias, (iv) conducta sexual de riesgo; y, (v) conductas violentas.

Todos los adolescentes están expuestos de una u otra forma al desarrollo de conductas de riesgo. Sin embargo, en consideración ciertas limitantes del presente estudio en cuanto a muestreo y elaboración y aplicación del instrumento, los datos obtenidos dejan cifras que pueden advertirse como signo de alarma en lo que respecta al consumo de sustancias, los comportamientos suicidas, y los trastornos de la conducta alimentaria y sedentarismo en los adolescentes; factores que sin duda pueden causar daños en la salud de los jóvenes a corto o largo plazo, logrando efectos negativos de alto impacto en la salud pública de los habitantes de la parroquia Ducur.

\section{Recomendaciones}

Se sugiere continuar las investigaciones referentes las conductas de riesgo para la salud del adolescente, en otras escuelas públicas y privadas, urbanas y rurales de tal forma que permita realizar un estudio comparativo, que sirva para la toma de decisiones frente a esta problemática social.

El trabajo mancomunado entre padres de familia, maestros y los propios adolescentes hará que se tome conciencia de la magnitud del problema antes que la prevalencia siga en aumento; para lo cual el desarrollo de talleres en asociación con instituciones involucradas garantizará el empoderamiento de todos los actores. La presencia de los padres de familia en esta etapa de la vida es trascendental, por lo que se recomienda fomentar la confianza y estar alerta a los comportamientos de los hijos adolescentes, sobre todo de aquellas conductas que puedan comprometer su integridad física y psicológica.

A las autoridades de los establecimientos educativos se sugiere poner énfasis en la vigilancia y control de conductas violentas que puedan presentarse dentro de la institución, Así mismo se hace un llamado a las autoridades de los gobiernos autónomos descentralizados para que fomenten la aplicación y/o construcción de políticas públicas, con la respectiva asignación de recursos, dirigidas a la prevención de comportamientos de riesgo en adolescentes, para precautelar la salud de este grupo vulnerable.

A los adolescentes se recomienda evitar comportamientos que puedan derivar en problemas de salud como anorexia, bulimia, pensamiento suicida, uso y abuso de alcohol, tabaco y drogas, embarazos no planificados o enfermedades de transmisión sexual; todo esto junto a estilos de vida saludables que incluyen la actividad física, dormir lo suficiente, evitar el estrés, entre otros.

\section{Fuente de Financiamiento}

Este estudio es autofinanciado.

\section{Conflicto de Intereses}

No existen conflictos personales, profesionales, financieroso de otro tipo.

\section{Consentimiento Informado}

Los autores cuentan con el consentimiento informado de los pacientes para la investigación, la publicación del caso y sus imágenes. 


\section{Referencias Bibliográficas}

1. García E, Muñoz N, Gaquín K, Hernández R. Conductas de riesgo en los adolescentes. Revista Cubanda de Medicina Militar. 2015;44(2):218-229.

2. Monteiro FN, Casotti CA, Alves A. Comportamento de risco á saúde de adolescentes escolares. Texto e Contexto Enfermagem. 2016;25(4):1-9.

3. Loarte MM. Cómo vivir con adolescentes. de políticas sociales y familia C, editor. Madrid: Comunidad de Madrid; 2016.

4. Villareal ME, Castro R, Domínguez R. Familia, adolescencia y escuela. 1st ed. UANL EU, editor. Monterrey: Universidad Autónoma de Nuevo León; 2016.

5. Organización Panamericana de la Salud. Estrategia y Plan de Acción Regional sobre Adolescentes y Jóvenes 20102018. 1st ed. OPS/OMS, editor. Washington: Organización Panamericana de la Salud; 2010.

6. Funes F, Gaete V. Conductas de riesgo en adolescentes con patología crónica compleja bajo control en un policlínico de un hospital pediátrico de Santiago. Revista Medica de Chile. 2016;144(6):734-742.

7. Díaz LC, Ramírez MT. Conductas problema en adolescentes en la ciudad de Monterrey, México. Enfermeria Global. 2014;13(1):1-17.

8. Organización Panamericana de la Salud/Organización Mundial de la Salud. Base de datos de mortalidad en la Región; 2016.

9. Caffe S, Dick B, Calle M, Díaz K. La salud de los adolescentes y jóvenes en la región de las Américas: Informe resumido; 2018.

10. Freire WB, Ramírez-Luzuriaga MJ, Belmont P, Mendieta MJ, Silva-Jaramillo K, Romero N, et al. Tomo I Encuesta Nacional de Salud y Nutrición: ENSANUT-ECU 2012. 1st ed. UNICEF, editor. Quito: Miniaterio de Salud Pública/Instituto Nacional de Estadística y Censos; 2014.

11. Freire WB, Belmont P, Rivas-Mariño G, Larrea A, Ramírez-Luzuriaga MJ, Silva-Jaramillo K, et al. Tomo II Encuesta Nacional de Salud y Nutrición: Salud Sexual y Reproductiva. 1st ed. Jara N, editor. Quito: Miniaterio de Salud Pública/Instituto Nacional de Estadística y Censos; 2015.

12. Argaez S, Echeverría R, Evia NM, Carrillo CD. Prevención de Factores de Riesgo en Adolescentes: Intervención para Padres y Madres. Psicologia Escolar e Educacional, SP. 2018;22(2):259-269.

13. Ordoñez PG, Barros SM. Normas y exigencias familiares con adolescentes que presentan problemas conductuales en UDIPSAI. Septiembre 2017- Enero 2018. Killkana
Salud y Bienestar. 2018;2(2):21-26.

14. Tena-Suck A, Castro-Martínez G, Marín-Navarrete R, Gómes-Romero P, Fuente-Marín A, Gómez-Martínez R. Consumo de sustancias en adolescentes: consideraciones para la práctica médica. Revista de Medicina Interna México. 2018;34(2):264-277.

15. Tegoma-Ruiz VM, Cortaza-Ramírez L. Prevalencia del consumo de alcohol en adolescentes de una secundaria de Coatzacoalcos, Veracruz. Enfermería Universitaria. 2016;13(4):239-245.

16. Da Silva SB. Comportamientos de riesgo en los adolescentes: Prevalencia y factores asociados; 2017.

17. Méndez J. Consumo de drogas e involucramiento parental entre estudiantes de secundaria de Costa Rica durante el 2015. Drugs and Addictive Behavior. 2017;2(2):193-204.

18. Vidal E, Hernández B. Conductas sexuales de riesgo asociadas a las infecciones de transmisión sexual en adolescentes de una comunidad. Revista Habanera de Ciencias Médicas. 2002;16(4):625-634.

9. Silva D, Valdivia M, Vicente B, Arévalo E, Dapelo R, Soto C. Intento de suicidio y factores de riesgo en una muestra de adolescentes escolarizados de Chile. Revista de Psicopatologia y Psicologia Clinica. 2017;22(1):33-42.

20. Fajardo E, Méndez C, Jauregui A. Prevalencia del riesgo de trastornos de la conducta alimentaria en una población de estudiantes de secundaria, Bogotá - Colombia. Revista Med. 2017;25(1):46-57.

21. Vivo S, Sarič D, Muñoz R, McCoy S, López-Peña P, Bautista-Arredondo S. Guía para medir comportamientos de riesgo en jóvenes. 1st ed. Vivo S, Sarič D, editors. Washington D. C.: Banco Interamericano de Desarrollo; 2013.

Recibido: 6 de mayo de 2019

Aceptado: 3 de septiembre de 2019 